\title{
Ecología y etnobotánica dos plantas comestibles de la comunidad Altoandina de Kcana Janansaya, distrito Kunturkanki, Provincia Canas, Cusco
}

\section{Ecology and ethnobotany of two species of edible plants of the high- Andes community Kcana Janansaya, district Kunturkanki, Province Canas, Cusco}

\section{Pascual Pacori-Gonzales}

Herbario Vargas (CUZ), Facultad de Ciencias Biológicas, Universidad Nacional de San Antonio Abad del Cusco. Prolongación Av. de la Cultura s/n, Cusco, Perú. fcm999@hotmail.com.

\section{F. Callañaupa-Mendoza, E. Callañaupa-Mendoza \& Y. Sánchez-Callañaupa}

Facultad Ing. Agroindustrial - Sicuani. Universidad Nacional de San Antonio Abad del Cusco.fcm999@hotmail.com

\section{Resumen}

El presente trabajo de investigación se realizó en la comunidad altoandina de Kcana Janansaya, distrito Kunturkanki, Provincia Canas, Región Cusco, a 4085 m de altitud, desde febrero a abril de año 2013; con el objetivo de estudiar dos especies de plantas comestibles de la comunidad mencionada. El método empleado fue el Muestreo Aleatorio Simple (MAS) al azar. El área de la muestra es de 0.135 ha $\left(1350 \mathrm{~m}^{2}\right)$ distribuidos en 9 parcelas rectangulares de $150 \mathrm{~m}^{2}(10 \times 15 \mathrm{~m})$, las que a su vez se dividieron en subparcelas de $1 \mathrm{~m}^{2}(1 \times 1 \mathrm{~m})$. Se registró 783 especímenes, de los cuales, la especie Stangea rhizantha (A. gray) Killip predomina con $50.83 \%$ (398 muestras), en comparación con la especie Hypochaeris taraxacoides (Walpers) Bentham \& Hooker f. que posee el $49.16 \%$ (385 muestras). De las cualidades nutricionales; la segunda especie resalta en carbohidrato con $18.0 \%$, fósforo $83.0 \mathrm{mg} / 100$ y potasio $14.0 \mathrm{mg} / 100$. De las 69 personas encuestadas, el $56.52 \%$ (21 varones, 18 mujeres) informan que ambas especies se consumen crudo, el $36.23 \%$ ( 15 varones, 10 mujeres) indican que se expenden en los mercados comunales y distritales, el $7.24 \%$ (4 varones, 1 mujer) no saben ni opinan.

Palabras claves: Especies, comunidades, muestras, encuesta, crudo.

\section{Abstract}

The present reseach was performed in the community altoandina of Kcana Janansaya, distrito Kunturkanki, Provincia Canas, Región Cusco, to 4085 m of altitude, from February to April in 2013; with the objective of study two species of plants edible of the community mentioned. The method employee is the Sampling Random Simple (MAS) at random. The area of the shows is of 0.135 ha $\left(1350 \mathrm{~m}^{2}\right)$ distributed in nine plots rectangular of $150 \mathrm{~m}^{2}\left(10 \times 15 \mathrm{~m}\right.$.), the what to its time were divided in subplots of $1 \mathrm{~m}^{2}(1 \times 1 \mathrm{~m})$. It recorded 783 specimens, of the which, the specie Stangea rhizantha (A. gray) Killip predominant with 50.83\% (398 samples), in comparison with the specie Hypochaeris taraxacoides (Walpers) Bentham \& Hooker f. that has the $49.16 \%$ (385 samples). The analysis bromatological the second species highlighted in carbohydrate with 18.0 $\%$, phosphorus $83.0 \mathrm{mg} / 100$ and potassium $14.0 \mathrm{mg} / 100$. Of the 69 peoples surveyeds the $56.52 \%(21$ males, 18 females) report what both species is consumed crude, the $36.23 \%$ (15 males, 10 females) indicate what is expend in the markets community and district, the $7.24 \%$ ( 4 males, 1 female) say they do not know

Keywords: Species, communities, samples, bromatological, survey, crude. 


\section{Introducción}

Las praderas altoandinas de la región del Cusco, poseen una flora nativa muy diversificada, la que más predomina son las gramíneas, herbáceas, arbustos y subarbustos, también están presentes las plantas alimenticias silvestres y que abunda en la estación lluviosa, la presencia de estas especies vegetales varían en proporciones diferenciables

Carrasco (1987), manifiesta que el estudio de la especie Stangea henrici Graebn, "chicuru" se llevó a cabo en el anexo Chañi del distrito de Condoroma, Provincia de Espinar - Cusco, se colectó ejemplares frescos libre de impurezas para el análisis bromatológico, cuyo resultado es como sigue: almidón $45.5 \%$, fibra neta 12.11 $\%$, proteínas $2.7 \%$.

Condori \& Cahuana (2000), mencionan que de los estudios realizados en la comunidad de Rosaspampa y Apacheta ubicados entre Ayacucho y Huancavelica, colectaron $2 \mathrm{~kg}$ de $S$, henrici, para el análisis bromatológico, los resultados obtenidos es como sigue: a) raíz: carbohidrato $18.7 \%$, ceniza $6.2 \%$, proteína 5.7 $\%$, grasa $4.7 \%$. b) hojas: carbohidrato $38.2 \%$, ceniza $12.3 \%$, proteína $8.7 \%$, grasa $4.7 \%$. La raíz es consumida en forma cruda y el sabor es dulcete-azucarado.

Huisa (2004), menciona que la especie $H$. taraxacoides (Walpers) Bentham \& Hooker f. "misq'i - pilli" y la especie H. sissiflora H.B.K. "jayac-pilli", son consumidas las partes blancas de las hojas en forma cruda, también se consumen la inflorescencia, el "jayac - pilli" tiene raíz alargada que contiene látex la que es masticado como chicle andino (kausillu) y los jóvenes del lugar usan con frecuencia, además estas especies son utilizados en infusión para el hígado (antibiloso) y cólicos intestinales.
Pestalozzi (2004), indica que la especie $\mathrm{H}$. taraxacoides es consumida las partes blancas de las hojas más la inflorescencia en forma cruda por los habitantes de las zonas altoandinas, la especie $H$. echegarayi tiene raíz alargada la que es utilizada como chicle andino (q'ausilla) y es muy apreciada por los jóvenes del lugar.

En la comunidad de Kcana Janansaya, la presencia de especies vegetales alimenticias silvestres abunda en la estación lluviosa y se encuentra asociada con otras especies, por tal razón, el presente trabajo de investigación se llevó a cabo con el objetivo de evaluar los recursos fitogenéticos alimenticios silvestres en la comunidad mencionada, para lo cual, se utilizó el Método de Muestreo Aleatorio Simple (MAS) al azar. Mediante el inventario se registró la composición cuantitativa de las especies colectadas, además se realizó el análisis bromatológico con el fin de conocer las cualidades nutricionales. De las personas encuestadas se recopiló valiosa información sobre la utilidad de algunas plantas. Los resultados obtenidos constituyen un aporte al conocimiento de la diversidad de especies alimenticias silvestres para la comunidad.

\section{Materiales y métodos}

\section{Área de estudio}

Políticamente la comunidad de Kcana Janansaya del distrito de Kunturkanki, se encuentra ubicada al Sur de la Provincia de Canas, Región Cusco. Geográficamente se encuentra ubicada a $14^{\circ} 30^{\prime} 30^{\prime \prime} \mathrm{LS}$ y $71^{\circ} 15^{\prime} 28^{\prime}$ " LW, a $4085 \mathrm{~m}$ de altitud; comprende una extensión de $1657.5 \mathrm{ha}\left(16.57 \mathrm{~km}^{2}\right)$.

El acceso a la comunidad mencionada es por carretera asfaltada de Cusco a Sicuani a una distancia de $138.73 \mathrm{~km}$. De Sicuani hasta el pueblo "El Descanso" es por carretera a $65 \mathrm{~km}$ - 
-de distancia De Descanso hasta la comunidad Kcana Janansaya es por trocha carrozable a $10 \mathrm{~km}$. de distancia.

El clima es frígido, la temperatura tiene una fuerte variabilidad diaria, hay momentos de sol, frío, sombra, nublado, viento; la noche es frígido, siendo la temperatura promedio anual de $11.6^{\circ} \mathrm{C}$, sin embargo, en época de helada (mayo - julio) disminuye considerablemente, bajando hasta $-9.7^{\circ} \mathrm{C}$ en el mes de julio. La precipitación total anual es de $717.16 \mathrm{~mm}$ (SENAMHI - Espinar, 2008 - 2013).

El área que cubre la zona de estudio se encuentra dentro de la Ecorregión Puna - Altos Andes y Páramo (Brack 2000), de acuerdo al Sistema de Clasificación de Zonas de Vida de Holdridge (1957), corresponde a las zonas de vida: bosque húmedo Montano Subtropical (bh - MS) y páramo muy húmedo Subalpino Subtropical (pmh - SaS).

\section{Metodología}

La presente investigación corresponde a un Nivel Aplicativo, que consiste en ponerse en contacto directo con la realidad a investigarse y con las personas que están relacionados con el lugar, por lo que, es necesario manipular y modificar de manera especial el área de estudio, seguidamente se aplicó el método científico que consiste en la observación directa de las especies vegetales alimenticias silvestres y de las plantas que se hallan asociadas, la hipótesis planteada, con la evaluación efectuada se obtiene resultados y conclusiones confiables.

\section{Muestreo poblacional}

El presente estudio se llevó a cabo desde el mes de febrero hasta abril del año 2013, la gradiente altitudinal de las especies determinadas comprende desde $4021 \mathrm{~m}$. hasta-
-4 392 m., de altitud, iniciándose en la llanura del sector Rumichaca, luego la ladera de Cebadilla Pucara, por ultimo el cerro Jatun Huaraconi, la distancia entre cada rango es de $150 \mathrm{~m}$. constituyéndose 3 rangos y en cada rango se muestrearon 3 parcelas, para lo cual, se utilizó el método de Muestreo Aleatorio Simple (MAS) al azar. El área de la muestra es de 0.135 ha $\left(1350 \mathrm{~m}^{2}\right)$ distribuidos en 9 parcelas rectangulares de $150 \mathrm{~m}^{2}$ (10 x $15 \mathrm{~m}$.), las que a su vez se dividieron en subparcelas de $1 \mathrm{~m}^{2}(1 \mathrm{x} 1$ m) con la finalidad de obtener un mejor muestreo.

Se colectaron de 3 a 4 muestras botánicas fértiles con flores y fruto por especie, tomando en cuenta el lugar de procedencia, hábitat, altitud, localidad, fecha de colección, las que fueron deshidratadas en el secadero del Herbario Vargas (CUZ). Se procedió a colocar las muestras en cartón secante y en aluminios corrugados, los cuales, se empilaron cuidadosamente, ya que de esto depende la presentación final de la muestra herborizada. Posteriormente al secado, se procedió a montar y etiquetar las mismas para su respectiva determinación con claves dicotómicas, bibliografía especializada, consulta a especialistas entendidos en la materia y comparación con las muestras patrón existente en el Herbario. Los especímenes determinados y herborizados (montaje) fueron depositados en el mismo Herbario

\section{Cualidades nutricionales}

Para conocer los valores nutricionales de las especies seleccionadas, se sometieron al análisis bromatológico mediante los métodos empleados en el Laboratorio química LAB Cusco (Figura 1). 


\section{Muestreo etnobotánico}

Se entrevistaron 69 personas entre varones $y$ mujeres de 18 a 70 ańos de edad, de acuerdo a los siguientes métodos: a) método de entrevista semiestructurada; que consiste en conversar y dialogar con personas de diferentes comunidades y con los habitantes de la población urbana. b) Método de flujo de información bilateral; que consiste en realizar una entrevista a las personas que viven en las mismas comunidades, sobre el conocimiento y uso de las plantas alimenticias silvestres. c) Método de juicio a expertos; que consiste en realizar una entrevista a las personas adultas mayores de edad, porque ellos adquirieron mucha experiencia durante toda su vida cotidiana y fueron quienes en realidad dieron una información precisa y valiosa de las formas de uso de las plantas alimenticias silvestres.

\section{Resultados}

En las áreas de estudio, se registró un total de 783 especímenes, de los cuales, la especie Stangea rhizantha predomina con 50.83\% (398 muestras), en comparación con la especie Hypochaeris taraxacoides que posee el $49.16 \%$ (385 muestras), (Tabla 1).

Tabla 1. Evaluación poblacional de especies.

\begin{tabular}{|l|l|l|l|}
\hline \multirow{2}{*}{ Familia } & \multirow{2}{*}{ Especie } & \multicolumn{2}{|l|}{ Comunidad Kcana Janansaya } \\
\cline { 3 - 4 } & & $(\%)$ & $\mathrm{N}^{\mathrm{P}}$ Muestras \\
\hline Caprifoliaceae. & Stangea rhizantha. & 50.83 & 398 \\
\hline Asteraceae. & Hypochaeris taraxacoides. & 49.16 & 385 \\
\hline Total. & \multicolumn{3}{|l}{783} \\
\hline
\end{tabular}

La posición taxonómica es de acuerdo al sistema de clasificación de A. Cronquist (1981).

Stangea rhizantha (A. Gray) Killip. (Caprifoliaceae).

Sinonimia: Stangea emiliae Graebner, ValerianarhizanthaA. Gray.

\section{Posición taxonómica}

Reino: Plantae

División : Magnoliophyta

Clase : Magnoliopsida (Dicotiledónea)

Subclase : Asteridae

Orden: Dipsacales

Familia: Caprifoliaceae (=Valerianaceae)

Género: Stangea.

Especie: Stangea rhizantha (A. Gray) Killip.

Nombre local : "chicuru"

Diagnosis de campo.- Plantas herbáceas anuales, de $4-7 \mathrm{~cm}$. de altura, acaule (postrada) en roseta basal. Raíces fusiformes que son mucho más engrosadas en su parte superior, esponjosas, blanquecinas, comestibles; tallos densamente cespitosos; hojas ovadas, bordes enteros o ligeramente crenados, carnosas, espatuladas. Limbo rugoso, glabra obtusa en la punta, de color verde olivo, de $1-2.5 \mathrm{~cm}$. de largo y $0.5-1 \mathrm{~cm}$. de ancho, peciolo corto de 5$10 \mathrm{~mm}$. de largo. Inflorescencia en forma de disco o en capítulo sésil, en el centro de la roseta de $1-2$ cm de diámetro, corola infundibuliforme a hipocratiforme de 4-5 $\mathrm{mm}$. de largo, pétalos blancos pentalobuladas, ovario sincárpico e ínfero, fruto en aquenio de 3 $\mathrm{mm}$. de largo con semillas estrechamente rectangulares.

Hábitat: crece en laderas y partes altas de la montaña (cerros), asociada con pajonales, en suelos de tierras negruzcas, pedregosas. Adaptada a la crioturbación por la forma de la raíz. Florece de enero a abril (Figura1).

Cualidades nutricionales.- Se efectuó con la finalidad de valorar las cualidades nutricionales, cuyo resultado es como sigue: carbohidrato $11.7 \%$, proteína $4.1 \%$, grasa 0.68 $\%$, fibra $0.43 \%$, ceniza $0.34 \%$, acidez $0.17 \%$, fósforo $3.0 \mathrm{mg} / 100$, potasio $7.0 \mathrm{mg} / 100$, humedad $82.6 \%$, pH: 6.7 
Hypochaeris taraxacoides (Walpers) Bentham \& Hooker f.(Asteraceae).

Sinonimia: Hypochaeris stenocephala Gray ex Wedd.) Kuntze.

Posición taxonómica

Reino: Plantae

División: Magnoliophyta

Clase: Magnoliopsida (Dicotiledónea)

Subclae: Asteridae

Orden: Asterales.

Familia: Asteraceae.

Género: Hypochaeris.

Especie: Hypochaeris taraxacoides (Walpers) Bentham \& Hooker $f$

Nombrelocal: "misq'i-pilli".

Diagnosis de campo.- Plantas herbáceas anuales, de $4-9 \mathrm{~cm}$. de altura, arrosetada, (pegada al suelo), postrada, raíz central alargada y gruesa de $4-8 \mathrm{~cm}$. de largo, tallo sésil, hojas basales arrosetadas o pegadas al suelo, formando una roseta, de formas lanceoladas a lineales, glabras, bordes dentados, lámina de 3 $7 \mathrm{~cm}$. de largo y de $2-3 \mathrm{~cm}$. de ancho. Peciolos blancos comestible de $3-5 \mathrm{~cm}$. de largo, involucro cilíndrico de más o menos $20 \mathrm{~mm}$. de alto por $6 \mathrm{~mm}$. de ancho, filarias glabras. Flor amarilla en capítulo ubicada al centro de la raíz solitaria. Pedúnculo floral hasta $3 \mathrm{~cm}$. de largo, posee alrededor brácteas de color blanco a veces jaspeado y al centro liguliformes

Hábitat: crece en laderas y cerros, asociada con gramíneas y herbáceas. Florece de enero a abril (Figura 1).

Cualidades nutricionales.- Se sometió al análisis con la finalidad de valorar sus cualidades nutricionales arrojando los siguientes resultados: carbohidrato con $18.0 \%$, proteína $2.80 \%$, grasa $0.19 \%$, fibra $0.28 \%$, ceniza 0.13 -
-\%, acidez $0.41 \%$, fósforo $83.0 \mathrm{mg} / 100$, potasio $14.0 \mathrm{mg} / 100$, humedad $78.60 \%$, pH: 6.7 (tabla 2).

Tabla 2. Análisis bromatológico de especies.

\begin{tabular}{|l|l|c|}
\hline N_pík crpmq & Stangea rhizantha & Hipochaeris taraxacoides \\
\hline r J 0 & 6.70 & 6.70 \\
\hline Humedad (\%) & 82.60 & 78.60 \\
\hline Grasa (\%) & 0.68 & 0.19 \\
\hline Acidez (\%) & 0.17 & 0.41 \\
\hline Fibra (\%) & 0.43 & 0.28 \\
\hline Ceniza (\%) & 0.34 & 0.13 \\
\hline Proteína (\%) & 4.10 & 2.80 \\
\hline Carbohidratos (\%) & 11.70 & 18.00 \\
\hline Fósforo (mg/100) & 3.00 & 83.00 \\
\hline Potasio (mg/100) & 7.00 & 14.00 \\
\hline
\end{tabular}

Etnobotánica. Se referirse a la diversidad biológica, cultural y costumbres de las comunidades campesinas, los habitantes del lugar no olvidan las costumbres ancestrales de las formas de uso de las especies vegetales que heredaron de una generación a otra, teniendo estas consideraciones se realizó la encuesta a 69 personas, de los cuales, el $56.52 \%$ (21 varones, 18 mujeres) informan que la especie $S$. rhizantha particularmente las raíces son consumidas por la población lugareña, cuando se consumen crudas se denominan "chawa chicuru"; sancochadas se denominan "chicuru yanuy", cuando son trozadas o picadas y se les agrega a la sopa de almuerzo se les conoce como "chicuru api". En época de escasez de alimento se consume como sustituto de la papa en sopas de almuerzo, en cambio, la especie $H$. taraxacoides son consumidas las partes blancas de la hoja más la flor en forma cruda. El 36.23\% (15 varones, 10 mujeres) indican que se expenden ambas especies en los mercados comunales y distritales, en ferias sabatinas y dominicales. El 7.24\% (4 varones, 1 mujer) no saben ni opinan de la utilidad de la especie estudiada. (Figura 2).

Medicinal: El chicuru cuando se consume crudo es efectivo para el tratamiento de riñones- 


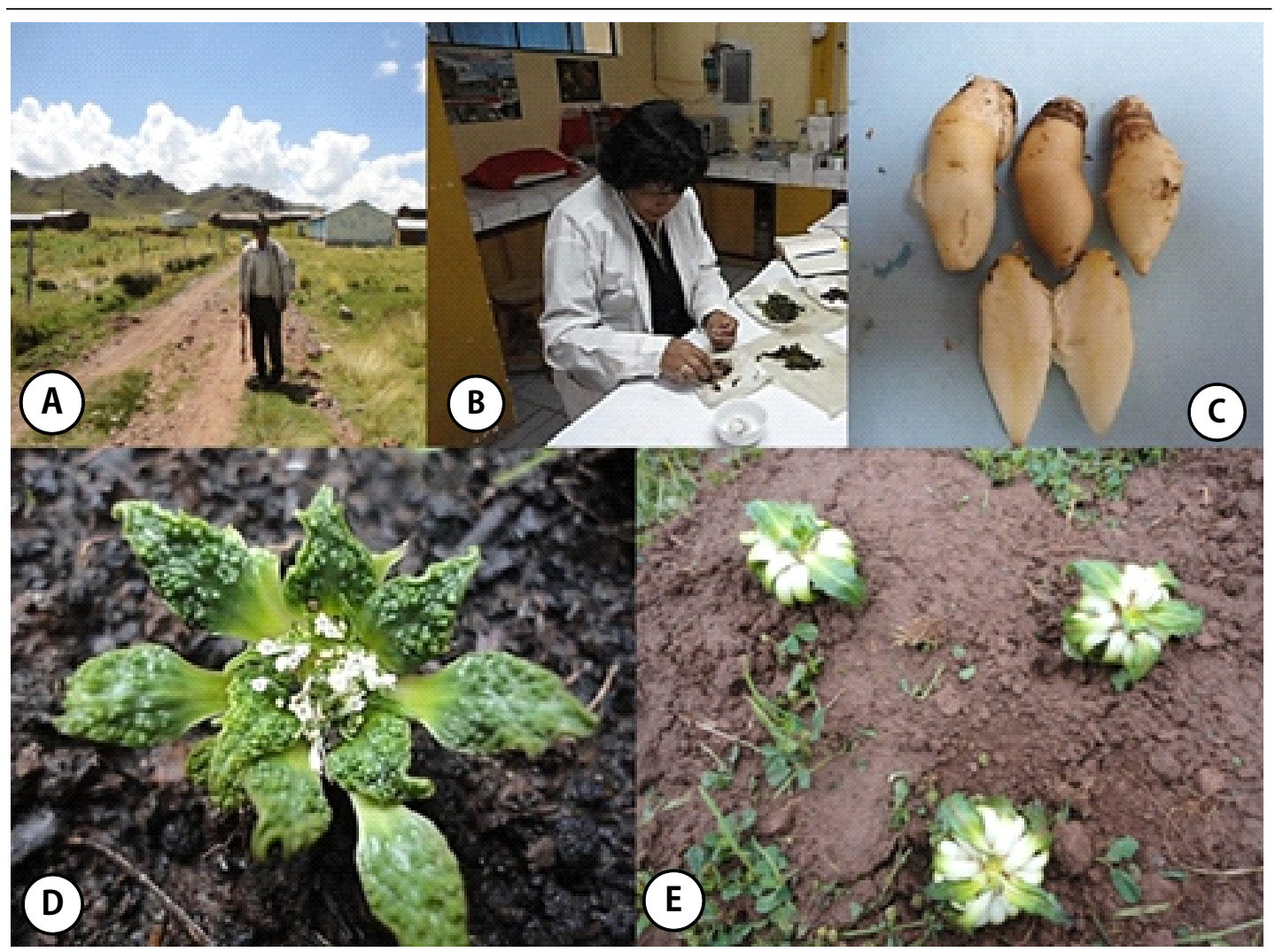

Figura 1. A. Comunidad Kcana Janansaya. B. Análisis, C. Raices y D, E. Hábito.

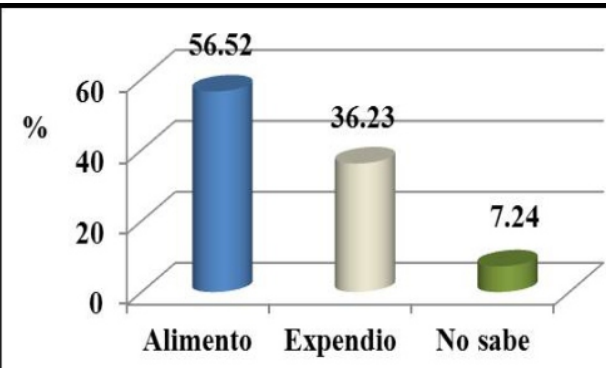

Stangea rhizantha e Hipochaeris taraxacoides.

Figura 2. Especies vegetales comestibles.

-y anemia, estimula el apetito sexual por contener zinc. El jugo de los frutos es fuente de energía (tónico) que alivia la tensión nerviosa. Las hojas en infusión para fiebre intestinal y malestares estomacales, en cambio, el misq'i- -pilli utilizan las raíces y hojas en infusión para el hígado (antibilioso).

Uso al pastoreo: la especie $S$. rhizantha es muy apetecida para ovinos y camélidos sudamericanos, las ovejas escarban la tierra con sus pezuñas hasta obtener las raíces de chicuru, las cuales, son consumidas por poseer alto valor nutritivo. Las hojas son consumidas por alpacas y llamas, los vacunos las consumen accidentalmente. La especie $H$. taraxacoides es muy palatable para ovinos, alpacas y llamas, los vacunos consumen accidentalmente.

Plantas asociadas.- Las especies vegetales comestibles se encuentran asociados con 15 familias, 34 géneros, 39 especies, la que más resalta es la familia Asteraceae con 13 géneros y 15 especies. (Tabla 3 ). 
Tabla 03. Listado de especies asociadas.

\begin{tabular}{|c|c|c|c|}
\hline Lo & Familias & Especies & Nombre local \\
\hline \multirow[t]{2}{*}{23} & \multirow[t]{2}{*}{ AMARANTHACEAE. } & Chenopodium murale Linnaeus. & purum - paicco. \\
\hline & & Gomphena meyeniana Walper. & puna-pimpinela. \\
\hline 02 & APOCYNACEAE. & Sarcostemma lysimachioides (Weddell.) R. Holm. & $\begin{array}{l}\text { llama - llama, } \\
\text { pampa lirija. }\end{array}$ \\
\hline \multirow[t]{15}{*}{03} & \multirow[t]{15}{*}{ ASTERACEAE. } & Baccharis incarum Wedd. & puna - chillca. \\
\hline & & Bidens andicola H. B. K. & sillkiwa, quico. \\
\hline & & Bidens triplinervia H. B. K. & sillkiwa, quico. \\
\hline & & $\begin{array}{l}\text { Chersodoma jodopappa } \\
\text { (Schultz - Bip.) Cabrera. }\end{array}$ & --- \\
\hline & & Galinsoga mandonii Schulttz - Bip. & $-\ldots$ \\
\hline & & $\begin{array}{l}\text { Gamochaeta americana } \\
\text { (Miller.) Weddell. }\end{array}$ & q'eto - q'eto. \\
\hline & & Grindelia boliviana Rusby. & chiri - chiri. \\
\hline & & $\begin{array}{l}\text { Paranephelius uniflorus } \\
\text { Poeppig. }\end{array}$ & ch'ahui - ch'ahui. \\
\hline & & Perezia multiflora (H. B. K.) Lessing. & chancorma. \\
\hline & & Senecio condimentarius Cabrera. & marancera. \\
\hline & & Senecio vulgaris $\mathrm{L}$. & purun - k`ana. \\
\hline & & Stevia mandonii Schultz - Bip. & chipi - chipi. \\
\hline & & Taraxacum officinale $\mathrm{F}$. H. Wigg. & pilli - pilli. \\
\hline & & Tanacetum vulgare Linnaeus. & palma real. \\
\hline & & Werneria orbignyana Wedd. & papusa. \\
\hline \multirow[t]{2}{*}{04} & \multirow[t]{2}{*}{ BRASSICACEAE. } & $\begin{array}{l}\text { Lepidium bipinnatifidum } \\
\text { Desvaux. }\end{array}$ & chichicara. \\
\hline & & Lepidium chichicara Desvaux. & chichicara. \\
\hline 05 & EPHEDRACEAE. & Ephedra rupestris Bentham. & puna pinco - pinco. \\
\hline 06 & EUPHORBIACEA & $\begin{array}{l}\text { Euphorbia raphanorrhiza } \\
\text { (Millspaugh.) J. F. Macbride. }\end{array}$ & huachanq'a. \\
\hline 07 & FABACEAE. & Lupinus aridulus C. P. Smith. & k'era, tarhui silvestre. \\
\hline 08 & GENTIANACEAE. & Gentianella scarlatina (Gil.) Zar. & q'ellofallcha. \\
\hline 09 & LAMIACEAE. & Lepichina meyenii (Walp.) Epling. & pacha salvia. \\
\hline 10 & MALVACEAE. & Acaulimalva engleriana (Urbrich) Kraporvickas. & altea. \\
\hline 11 & OXALIDADEAE. & Oxalis oreocharis Diels. & atoc - occa. \\
\hline \multirow[t]{8}{*}{12} & \multirow[t]{8}{*}{ POACEAE. } & Aciachne aciculares Laegaard. & paku paku. \\
\hline & & Agrostis tolucensis H. B. K. & $-\cdots$ \\
\hline & & Bromus catharticus M. Vahl. & cebadilla. \\
\hline & & Calamagrostis heterophylla (Wedd.) Pilger. & -- \\
\hline & & Calamagrostis vicunarum. & $-\cdots$ \\
\hline & & Festuca dolichophylla J. Presl. & ccoya, chillihua. \\
\hline & & Hordeum muticum J. Presl. & jucuchac-chupan. \\
\hline & & Stipa ichu (Ruiz \& Pav.) Kunth. & k’quisi ichu. \\
\hline 13 & POLYGONACEAE. & Muehlenberckia volcánica (Benth.) Endl. & mullaq'a. \\
\hline 14 & ROSACEAE & Alchemilla pinnata Ruiz \& Pavon. & sillu - sillu. \\
\hline \multirow[t]{2}{*}{15} & \multirow[t]{2}{*}{ URTICACEAE. } & Urtica flabellata Kunth. & curo quisa. \\
\hline & & Urtica magellanica Juss ex Paiv. & mula quisa, \\
\hline
\end{tabular}




\section{Discusión}

El presente estudio da a conocer que la especie Stangea rhizantha presenta valores nutricionales en carbohidrato con $11.7 \%$, proteína $4.1 \%$, y fibra $0.43 \%$, sin embargo, Carrasco (1987), manifiesta que la especie Stangea henrici contiene almidón el $45.5 \%$, proteína $2.7 \%$ y fibra neta $2.11 \%$, ambos autores demuestran que los resultados varían en proporciones diferenciables.

\section{Conclusiones}

Se registró 783 especímenes, de los cuales, la especie Stangea rhizantha predomina con 50.83 \% (398 muestras), en comparación con la especie Hypochaeris taraxacoides que posee el $49.16 \%$ (385 muestras).

Las especies vegetales comestibles se encuentran asociados con 15 familias, 34 géneros, 39 especies, la que más resalta es la familia Asteraceae con 13 géneros y 15 especies.

La especie $H$. taraxacoides posee alto valor nutricional en carbohidrato con $18.0 \%$, fósforo $83.0 \mathrm{mg} / 100$ y potasio $14.0 \mathrm{mg} / 100$ en comparación con la especie Stangea rhizantha que posee menor valor nutricional.

De las 69 personas encuestadas, el $56.52 \%$ (21 varones, 18 mujeres) informan que la especie Stangea rhizantha, se consume la raíz en forma cruda, en cambio, la especie Hipochaeris taraxacoides, se consume crudo las partes blancas de la hoja, también se consume la inflorescencia. El $36.23 \%$ (15 varones, 10 mujeres) indican que se expenden ambas especies en los mercados comunales y distritales. El 7.24\% ( 4 varones, 1 mujer) no saben ni opinan de la utilidad de la especie estudiada.

\section{Sugerencias}

Profundizar trabajos de investigación más específicos sobre plantas alimenticias silvestres, para conservar la diversidad genética de la especie nativa altoandina en otras zonas.

Evitar la degradación y desequilibrio del ecosistema, en especial las áreas que corresponde al hábitat de planicies, laderas y cumbres altas, por el sobrepastoreo de ganado, para conservar y evitar su extinción en el tiempo.

Difundir la existencia y la importancia de las especies estudiadas entre los pobladores urbanos, por sus cualidades nutricionales.

\section{Agradecimientos}

Se les agradece infinitamente al Sr. Luciano Arapa Quispe y su esposa Sra. Luciana Cárdenas Lozano, quienes de una u otra forma nos brindaron su valiosa colaboración. Así mismo al Sr Eustaquio Hanco Mamani, quien participó como asistente de campo en la identificación de las plantas con nombres locales y por las facilidades de hospedaje que nos brindó incondicionalmente.

\section{Literatura citada}

Brack, E. A. 2000. Ecologia del Perù. Edit. Bruño. Lima-Perú. pp. 83-89

Brack, E. A. 2000. Ecorregiones del Perú [En línea, r e c u p e r a d o m a y o $\left.20 \begin{array}{lllllll} & 0 & 1 & 1\end{array}\right]$ : http://www.slideshare.net/jorgeolaya/ecorregio n e s - d e l - p e r u , http://www.peruroutes.com/peru_ecologia.htm Carrasco, A. A. (1987). Estudio Botánico e Importancia en la Alimentación del "chiquro" Stangea henrici Graebn. Seminario. Fac. Biología -UNSAAC. Cusco-Perú. pp. 8-10, 13.

Condori \& Cahuana. (2000). Estudio nutricional de Stangea rhizanta (Chicuro) y su aprovechamiento. Edit. s/e. Ayacucho - Perú. pp. $2-15$. 
Huisa, J. T. 2004. Selección de pastos naturales en los andes del Sur del Perú: Centro Experimental "La Raya" UNSAAC. Edit. Universitaria-UNSAAC. Cusco-Perú.

Pestalozzi, H. U. 2004. Flora ilustrada alto andina. Edit. ODEC. Cochabamba Bolivia. pp. 62, 65-66. 
Pacori et al.: Ecología y etnobotánica de plantas comestibles en la Comunidad de Kcana Janansaya, Cusco 